\title{
REVIEW
}

\section{Chlamydia pneumoniae and atherosclerosis}

\author{
Y-K Wong, P J Gallagher, M E Ward
}

Wessex Cardiothoracic Unit, Southampton General Hospital, Tremona Road, Southampton, Hampshire SO16 6YD, UK

Y-K Wong

Department of Pathology, Southampton General Hospital,

Southampton, UK

P J Gallagher

Molecular

Microbiology,

Southampton

University Medical

School, Southampton

General Hospital,

Southampton, UK

ME Ward

Correspondence to: Dr Yuk-ki Wong.

email: YW2@soton.ac.uk

Accepted for publication 20 October 1998

\begin{abstract}
Objective-To review the literature for evidence that chronic infection with Chlamydia pneumoniae is associated with atherosclerosis and acute coronary syndromes.
\end{abstract}

Data sources-MEDLINE and Institute of Science and Information bibliographic databases were searched at the end of September 1998. Indexing terms used were chlamydi ${ }^{\star}$, heart, coronary, and atherosclerosis. Serological and pathological studies published as papers in any language since 1988 or abstracts since 1997 were selected.

Data extraction-It was assumed that chronic $C$ pneumoniae infection is characterised by the presence of both specific IgG and IgA, and serological studies were examined for associations that fulfilled these criteria. Pathological studies were also reviewed for evidence that the presence of $C$ pneumoniae in diseased vessels is associated with the severity and extent of atherosclerosis.

Data synthesis-The majority of serological studies have shown an association between $C$ pneumoniae and atherosclerosis. However, the number of cases in studies that have reported a positive association when using strict criteria for chronic infection is similar to the number of cases in studies which found no association. Nevertheless, the organism is widely found athough it may not be at all diseased sites and is not confined to the most severe lesions. Rabbit models and preliminary antibiotic trials suggest that the organism might exacerbate atherosclerosis.

Conclusion-More evidence is required before $C$ pneumoniae can be accepted as playing a role in atherosclerosis. Although use of antibiotics in routine practice is not justified, large scale trials in progress will help to elucidate the role of $\boldsymbol{C}$ pneumoniae. (Heart 1999;81:232-238)

The organism now known as Chlamydia pneumoniae was first isolated in 1965 in Taiwan. It was subsequently recognised to be a cause of acute respiratory disease ${ }^{1}$ giving rise to the acronym TWAR (Taiwan acute respiratory) agent. Chlamydia are unique, obligate, intracellular bacterial pathogens of eukaryotic cells. As they are difficult organisms to culture, much knowledge about $C$ pneumoniae infections has come from serological studies, many of which, since 1988, have shown an association between $C$ pneumoniae infection and atherosclerosis. However, association does not prove causality and, unfortunately, serological distinction between past and current infection is difficult.

In a general population, infection was absent in children under 5 years, attaining a peak incidence of $9.2 \%$ in those aged $5-10$ years before falling to $1.5 \%$ over the age of $20 .^{2}$ By the age of 20 years, prevalence of specific IgG has already reached $50 \%$, persisting or even increasing with age. ${ }^{3-5}$ Seroprevalence is proportional to the product of annual incidence and the duration of seropositivity following infection. As specific IgG antibodies are thought to disappear about three years following primary $C$ pneumoniae infection, ${ }^{6}$ an annual incidence of $1.5 \%$ should result in a prevalence of $4.5 \%$ rather than the observed $50 \%$. One possibility is that there is a high prevalence of chronic infection driving a persistent specific IgG response. This suggests that the optimal time to eradicate $C$ pneumoniae infection is around the age of 20 years, when half the population is seropositive and after which the risk of reinfection is relatively low. However, epidemics do occur ${ }^{7}$ and IgG titres probably decay more slowly than previously thought. Of 90 students followed up for up to five years, only those with an initial low titre of $1: 16$ had a geometric mean titre of less than this at five years. ${ }^{6}$ Therefore, a high seroprevalence might indicate a population characterised not by chronic infection needing treatment but by a high past exposure.

The problem is how to prove persisting infection against a background of persisting antibody. In primary respiratory infection, the predominant $\operatorname{IgM}$ response is followed by a delayed IgG and a delayed, weak, or absent IgA response. Adult infections are thought to be mainly reinfections characterised by specific IgG and IgA antibody responses without changes in IgM. ${ }^{9}$ Thus, accurate detection of acute infection requires paired samples taken four to six weeks apart and the demonstration of a threefold or more rising titre. Serological criteria for chronic infection are more controversial but persistently raised specific IgA and 
IgG is generally accepted as an indication of chronic infection, since IgA is thought to have a much shorter half life than IgG. These uncertainties partly explain why different authors have used different criteria for seropositivity. The picture is further complicated by reports of acute infection without an antibody response, ${ }^{10} 11$ and that few studies of antibody response have used culture and antigen or DNA detection as absolute criteria of current infection. Compounding these problems, serology uses crude, whole $C$ pneumoniae antigens, containing epitopes potentially cross reactive with antibodies to other
Chlamydia species, ${ }^{12}$ to other Gram negative bacteria, ${ }^{13}$ or even to human heat shock protein as has been described for Chlamydia trachomatis. ${ }^{14}$ Atherosclerosis is known to be associated with the presence of antibody to human heat shock protein, ${ }^{15}$ and the possible confounding effects of such antibody has not been determined in any serological study of $C$ pneumoniae and heart disease.

Pathological studies have generally shown that $C$ pneumoniae is more common in atherosclerotic than control blood vessels. However, such studies do not show whether infection preceded or followed the development of

Table 1 Studies investigating the serological association between $C$ pneumoniae and atherosclerosis

\begin{tabular}{|c|c|c|c|c|c|}
\hline \multirow[b]{2}{*}{ Study } & \multirow[b]{2}{*}{ Case control* } & \multicolumn{3}{|l|}{ Association witht } & \multirow[b]{2}{*}{ Diagnostic antibody titre $\neq$} \\
\hline & & $\operatorname{Ig} G$ & $\operatorname{Ig} A$ & ICs & \\
\hline \multicolumn{6}{|l|}{ Prospective studies } \\
\hline \multirow[t]{2}{*}{ Saikku $(1992)^{19}$} & $103 v 103$ & \multicolumn{3}{|c|}{$2.2(1.1-4.5) \quad 2.6(1.2-5.2)$} & $\operatorname{IgG} \geqslant 128$ and $/$ or IC or IgA $\geqslant 64$ \\
\hline & Cardiac death or MI & \multicolumn{3}{|c|}{6 months before event but not 5 years before } & and/or IC \\
\hline \multirow[t]{3}{*}{ Miettinen $(1996)^{20}$} & $162 v 636$ (NIDDM) & \multirow{2}{*}{\multicolumn{3}{|c|}{$32 \% v 15 \%$}} & $\operatorname{IgG} \geqslant 128$ and $\operatorname{Ig} A \geqslant 40$ \\
\hline & $40 v 1155$ (No DM) & $2.44(0.98-6.08)$ & & & \\
\hline & Cardiac death or MI & \multicolumn{3}{|c|}{ Only in non-diabetic men in East Finland } & \\
\hline \multirow[t]{2}{*}{ Ossewaarde $(1998)^{29}$} & $54 v 108$ & $52 \%$ v $34 \%$ & No & No & ELISA \\
\hline & Cardiac death or MI or angina & \multicolumn{3}{|l|}{$2.8(1.3-5.8)$} & \\
\hline \multirow[t]{2}{*}{ Nieto $(1997)^{30}$} & $256 v 550$ & \multirow{2}{*}{\multicolumn{3}{|c|}{ No }} & $\operatorname{IgG} \geqslant 64$ \\
\hline & $\begin{array}{l}\text { Cardiac death, } \mathrm{MI} \text { or } \\
\text { revascularisation }\end{array}$ & & & & \\
\hline \multirow[t]{2}{*}{ Siscovick $(1998)^{31}$} & $100 v 183$ & \multirow{2}{*}{\multicolumn{3}{|c|}{ No }} & Not stated (abstract) \\
\hline & Cardiac death or MI & & & & \\
\hline Cross sectional studies & & & & & \\
\hline Diedrichs $(1997)^{21}$ & $\begin{array}{l}131 v 63 \\
\mathrm{CA}\end{array}$ & $66 \% v 48 \%$ & $44 \%$ v $22 \%$ & & \\
\hline Halme $(1997)^{22}$ & $93 \approx 115$ & $100 \% v 61 \%$ (in $\mathrm{me}$ & & No & $\operatorname{IgG} \geqslant 128$ and $/$ or $\operatorname{Ig} A \geqslant 40$ \\
\hline Saikku $(1988)^{23}$ & $\begin{array}{l}\text { CA } \\
70 v 41\end{array}$ & $49 \%$ v $15 \%$ & $41 \%$ v $10 \%$ & & $\operatorname{IgG} \geqslant 128 \operatorname{Ig} A \geqslant 32$ \\
\hline & MI or stable angina & $5.5(2.1-14.7)$ & 417001070 & & $\lg G=128 \lg A=32$ \\
\hline Cook $(1998)^{24}$ & $176 v 1518$ & Acute infection 13.6 & & & $\operatorname{IgG} \geqslant 512$ or 4 fold $\operatorname{IgG}$ rise or \\
\hline & CVA & $4.2(2.5-7.1)$ & & & $\operatorname{Ig} M \geqslant 8$ \\
\hline & & Chronic infection 32 & $12.7 \%$ & & $\operatorname{IgG} \geqslant 64$ and $\leqslant 256$ or $\operatorname{Ig} A \geqslant 8$ \\
\hline & & $4.4(3-6.5)$ & & & \\
\hline Mazzoli $(1998)^{25}$ & $29 v 74$ & $82 \%$ v $34 \%$ & $71 \%$ v $14.9 \%$ & & \\
\hline & MI & & & & \\
\hline Dahlen $(1995)^{32}$ & $60 v 60$ & $93.3 \%$ v $78.3 \%$ & No & & $\mathrm{IgG} \geqslant 32$ \\
\hline & & $3.56(1-16.1)$ & & & $\operatorname{Ig} A \geqslant 16$ \\
\hline Mendall $(1995)^{33}$ & $100 v 64$ & $22 \% \vee 5 \%$ & No & & $\mathrm{IgG} \geqslant 64$ \\
\hline & $\mathrm{CA}$ & $7.4(1.7-33.1)$ & & & IgA not stated \\
\hline Blasi $(1997)^{28}$ & $61 v 61$ & $57 \%$ v $30 \%$ & No & & $\operatorname{IgG} \geqslant 16$ \\
\hline & MI & $3.2(1.5-6.8)$ & & & $\operatorname{Ig} A \geqslant 16$ \\
\hline Thom $(1991)^{34}$ & $461 v 95$ & $22 \%$ v $13 \%$ & & & $\mathrm{IgG} \geqslant 64$ \\
\hline & CA & $2(1-4)$ & & & \\
\hline Thom $(1992)^{35}$ & $171 v 120$ & $67 \%$ v $56 \%$ & & & $\operatorname{IgG} \geqslant 8$ \\
\hline & $\mathrm{CA}$ & $2.6(1.4-4.8)$ & & & \\
\hline Melnick $(1993)^{36}$ & 326 matched pairs. & $73 \%$ v $63 \%$ & & & $\operatorname{IgG} \geqslant 8$ \\
\hline & Carotid doppler & $2(1.2-3.4)$ & & & \\
\hline Patel $(1995)^{37}$ & $83 v 305$ & $30 \%$ v $18 \%$ & & & $\operatorname{IgG} \geqslant 64$ \\
\hline & Rose questionaire, ECG & $2.25(1.1-4.6)$ & & & \\
\hline Thomas $(1997)^{38}$ & 83 v 93 & $71.1 \%$ v $31.2 \%$ & & & $\operatorname{IgG} \geqslant 16$ \\
\hline & MI/IHD & $5.4(2.7-10.9)$ & & & \\
\hline Toss $(1998)^{39}$ & $256 v 190$ & Seroprevalence not & $36 \%$ ข $19 \%$ & & $\mathrm{IgG} \geqslant 16$ \\
\hline & Unstable angina & reported & & & $\operatorname{Ig} A \geqslant 64$ \\
\hline Wimmer $(1996)^{40}$ & $58 v 52$ & No & $47 \%$ v $23 \%$ & $24 \%$ v $8 \%$ & $\operatorname{IgG} \geqslant 32$ \\
\hline & CVA & & $1.7(1.1-2.7)$ & $2(1.1-3.8)$ & $\operatorname{Ig} A \geqslant 16$ \\
\hline Leinonen $(1990)^{16}$ & $42 v 41$ & No & No & $57 \%$ v $12 \%$ & $\operatorname{IgG} \geqslant 128$ \\
\hline & MI & & & $10(3-29)$ & $\operatorname{Ig} A \geqslant 32$ \\
\hline Leinonen $(1994)^{17}$ & $95 v 139$ & No & No & $58 \%$ v $26 \%$ & IgG $\geqslant 32$ and $\leqslant 128$ \\
\hline & MI & & & $4(2-7)$ & $\operatorname{Ig} A \geqslant 8$ and $\leqslant 32$ \\
\hline Weiss $(1996)^{18}$ & $65 v 28$ & No & No & & All titres considered \\
\hline & CA & & & & \\
\hline Kark $(1997)^{26}$ & $302 v 486$ & No & No & No & All titres considered \\
\hline & MI & & & & \\
\hline Boman $(1998)^{27}$ & $101 v 52$ & No & No & & \\
\hline Linnanmaki $(1993)^{41}$ & $46 v 46$ & No & & $41 \%$ v $15 \%$ & $\mathrm{IgG} \geqslant 32$ \\
\hline & CA & & & $4(1.4-11)$ & \\
\hline Anderson $(1998)^{42}$ & $124 v 97$ & No & & & $\mathrm{IgG} \geqslant 16$ \\
\hline & CA & & & & \\
\hline
\end{tabular}

^Number of cases and controls in final analysis and diagnosis of cases.

$\dagger$ Percentage of cases and controls who are seropositive or who have immune complexes (ICs). Odds ratio and $95 \%$ confidence interval. Adjusted figures are shown where reported by the authors.

$\ddagger$ Microimmunofluorescence test unless indicated

CA, coronary arteriograms; CVA, cerebrovascular accident; MI, myocardial infarction; IHD, ischaemic heart disease; NIDDM, non-insulin dependent diabetes mellitus; DM, diabetes mellitus; ELISA, enzyme linked immunosorbent assay. 

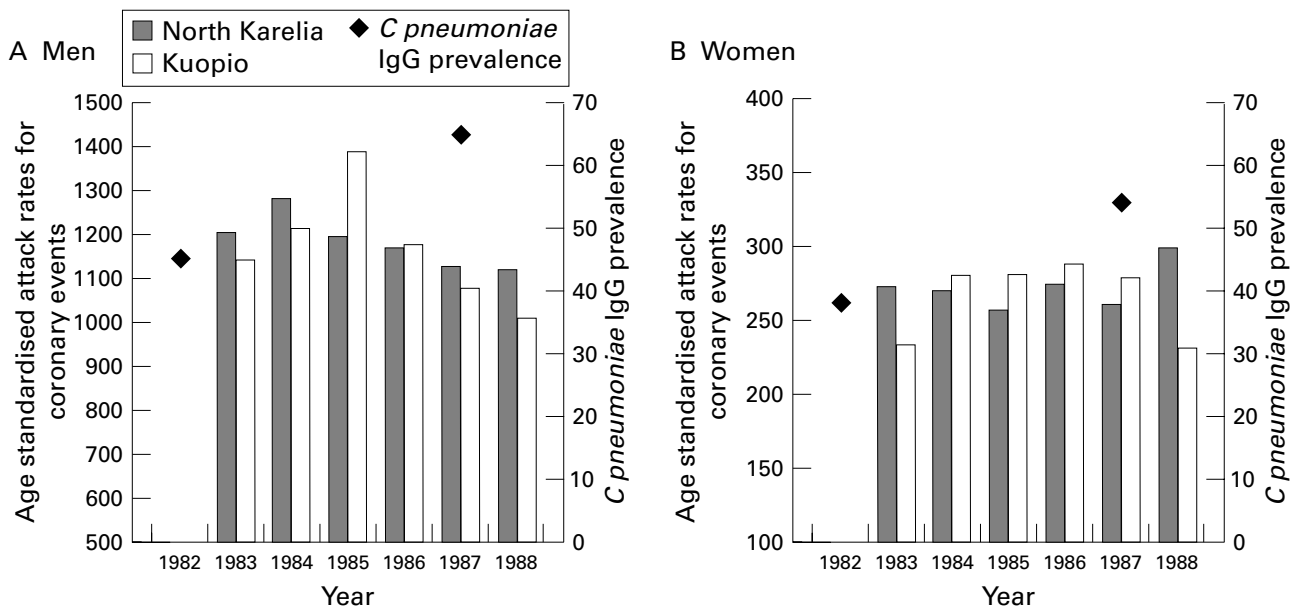

Figure 1 Age standardised attack rates per 100000 population in men (A) and women (B) for coronary events (definite and probable myocardial infarction and coronary deaths) in two regions of east Finland and prevalence of $C$ pneumoniae IgG.

atherosclerosis. Nevertheless, it might be expected that the distribution of $C$ pneumoniae should be related either to the extent or severity of atherosclerosis.

\section{Data sources and data extraction}

Serological and pathological studies published as papers in any language since 1988 or as abstracts since 1997 were searched for using the MEDLINE and Institute of Science and Information bibliographic databases. The search was completed at the end of September 1998. Indexing terms used were chlamydi ${ }^{\star}$ and heart, chlamydi ${ }^{\star}$ and coronary, and chlamydi ${ }^{\star}$ and atherosclerosis.

Information abstracted from serological studies included how many immunoglobulin classes were detected for and whether an association with atherosclerosis was found. Pathological studies were examined for information on the extent and distribution of $C$ pneumoniae in subjects with atherosclerosis.

\section{Serological studies of $C$ pneumoniae and atherosclerosis}

Table 1 shows that 21 of $27^{16-42}$ studies that have investigated $C$ pneumoniae and atherosclerosis have reported some sort of positive serological association. However, although all studies measured IgG, only three prospective and 15 cross sectional studies measured IgA. Of the cross sectional studies, five found an association with both immunoglobulins, three with IgG alone, and two with IgA alone. Five studies found no association with either immunoglobulin, although three of these did show an association with circulating chlamydial immune complexes. ${ }^{16}{ }^{17} 40$ Therefore, the serological evidence that chronic rather than past $C$ pneumoniae infection is associated with atherosclerosis is not compelling. In fact, the number of cases in studies which found a positive association with both immunoglobulins ${ }^{19-25}$ is similar to that in studies which found no association with either, ${ }^{16-18} 202627$ although controls were numerically far greater in the former group (642 and $3069 v 767$ and 1382). People with chronic debilitating diseases including heart failure are predisposed to respiratory tract infections and this may be one reason why people with coronary artery disease are more likely to have experienced $C$ pneumoniae infection. Controlling for known confounding factors such as smoking has not always been adequate, ${ }^{43}$ and it is not known whether there is an association of $C$ pneumoniae with heart failure. Of the five prospective studies, three reported a positive association but two of these depended on subset analysis. In one study, seropositivity was associated with coronary events six months but not five years before an event, ${ }^{19}$ while in the other an association was seen in non-diabetic men in east but not west Finland and not in diabetic men. ${ }^{20}$

Acute infections are easier to diagnose and four studies investigated whether antibody titre rises were associated with acute vascular events such as myocardial infarction and cerebrovascular accident. Cook and colleagues ${ }^{24}$ found an association with cerebrovascular accidents while Saikku and colleagues ${ }^{23}$ found an association between rising IgM titres to chlamydial group lipopolysaccharide and myocardial infarction. Blasi and colleagues ${ }^{28}$ found that $20 \%$ of patients with myocardial infarction had a rise in IgG but the proportion in the control group was unknown. No association was seen in the fourth study. ${ }^{16}$ Therefore, there is some evidence that acute infection is associated with acute vascular events. If this is true, the incidence of such events should increase during epidemics. East Finland has one of the highest coronary mortality rates in the world but there has been a gradual decrease since the 1970 s because of a primary prevention programme. East Finland has also contributed to the World Health Organisation monitoring of trends and determinants in cardiovascular disease (WHO MONICA) project, ${ }^{44}$ and serum samples from this project have been tested to estimate the population prevalence of $C$ pneumoniae. ${ }^{5}$ Figure 1 shows the attack rates of coronary events in two regions of east Finland and the seroprevalence of $C$ pneumoniae. It can be seen that in the epidemic year of 1987, acute coronary events did not increase. 


\section{Pathological studies of $C$ pneumoniae in blood vessels}

Techniques such as immunocytochemistry (ICC), the polymerase chain reaction (PCR), and culture have provided direct evidence that $C$ pneumoniae localises to blood vessels. Generally, ICC finds more evidence for $C$ pneumoniae than PCR but it is not known whether this is because of better sensitivity or worse specificity. It is notoriously difficult to obtain perfect ICC with severe atherosclerosis and the concern is that antibodies used in ICC cross react with components of atherosclerotic tissue. However, atherosclerotic tissue is known to contain inhibitors of PCR. These uncertainties make it difficult to estimate the prevalence of $C$ pneumoniae in blood vessels, especially as specimens positive by one technique were not necessarily positive by another. ${ }^{45}$ Culture of $C$ pneumoniae from blood vessels is technically challenging and had only been managed on two occasions ${ }^{46}{ }^{47}$ until recently when, following multiple serial passage, the organism was isolated from 11 of 70 atheromatous samples. ${ }^{48}$

Histological evidence of atherosclerosis is ubiquitous $^{49}$ and 11 of 25 studies $^{18}{ }^{45-48}$ 50-69 $^{\text {did }}$ not have control vessels (table 2). Only three studies had controls completely matched for age, origin of tissue, and tests used to detect for $C$ pneumoniae. Even then, control arteries either had histological evidence of early atherosclerosis, ${ }^{50}$ or came from arteries where adjacent segments had disease. ${ }^{51}$ For the other studies, control vessels tended to be numerically smaller and obtained at necropsy from younger subjects.
Although three studies failed to find evidence for $C$ pneumoniae in atherosclerotic vessels, ${ }^{18} 5253$ most have found it in $15-100 \%$ of cases. In contrast, it appears to be uncommon in control vessels and only in two studies was $C$ pneumoniae as prevalent in control as in diseased vessels. ${ }^{54}{ }^{55}$ In reality, it is likely that most control vessels, although macroscopically normal, did have early histological disease, which would suggest that $C$ pneumoniae is more common in people with severe rather than mild atherosclerosis. There were five studies in which the severity of atherosclerosis was formally graded on a histological basis. In one, $C$ pneumoniae was detected in $86 \%$ of severe compared with $6 \%$ of mild lesions using ICC. ${ }^{45}$ However, when a subset of samples was tested by PCR, discordant results were obtained and the prevalence in non-atherosclerotic or minimally atherosclerotic lesions was said to be quite high. ${ }^{70}$ In a necropsy study of 60 Alaskan natives who died mainly from noncardiovascular causes (mean age 34.1 years), the Stary classification was used to grade the severity of a segment of the right coronary artery obtained from each subject. ${ }^{56}$ Twenty two subjects were found to have $C$ pneumoniae but there was no difference in the severity of their coronary artery segments compared with that of subjects without $C$ pneumoniae. In total, 14 of 40 specimens with raised lesions were positive for $C$ pneumoniae compared with 7 of 18 specimens with flat lesions. The main interest of this paper was the finding that a high IgG titre of $\geqslant 256$ on average eight years before death was associated with the presence of $C$ pneumoniae in coronary arteries, suggesting

Table 2 Studies investigating the presence of $C$ pneumoniae in blood vessels

\begin{tabular}{|c|c|c|c|c|}
\hline \multirow[b]{2}{*}{ Study } & \multirow[b]{2}{*}{ Number of cases/controls ${ }^{*}$ (description of case tissue) } & \multicolumn{3}{|c|}{ Case and controls positive for $C$ pneumonia ${ }^{\star}$} \\
\hline & & PCR & ICC & Other \\
\hline \multicolumn{5}{|c|}{ Controlled studies. Age, tissue, and diagnostic method matched } \\
\hline Kuo $(1995)^{51}$ & $18 v 31$ (coronary artery) & $17 \% v 0 \%$ & $39 \% v 0 \%$ & \\
\hline Maass $(1997)^{50}$ & 61 v 39 (carotid endarterectomy) & $15 \% v 0 \%$ & & \\
\hline Petersen $(1998)^{61}$ & $40 v 40$ (AAA) & $35 \%$ v $5 \%$ & & \\
\hline \multicolumn{5}{|l|}{ Other controls } \\
\hline \multirow[t]{2}{*}{ Grayston $\dagger(1995)^{62}$} & $5 v 0$ (carotid endarterectomy) & $60 \%$ & $100 \%$ & \\
\hline & $56 v 6$ (archival or necropsy carotid specimens) & & $57 \%$ v $0 \%$ & \\
\hline Ongt $(1995)^{55}$ & 32 AAA repairs $v 6$ patients with normal vascular tissue & $44 \% v 50 \%$ & $3 / 8(38 \%) v$ not done & \\
\hline Jackson $(1997)^{63}$ & $38 v 38$ (vascular $v$ non-vascular tissue from necropsy cases) & $16 \% v 0-8 \%$ & $24 \%$ v $5-11 \%$ & \\
\hline Juvonen $(1997)^{64}$ & $12 v 9$ (abdominal aortic aneurysm) & $6 / 6 v 0 / 9$ & $100 \%$ v $0 \%$ & \\
\hline Kuot $(1997)^{65}$ & $23 v 8$ (diseased femoral and popliteal arteries) & $48 \%$ v $0 \%$ & & $0 \% \neq v$ not done \\
\hline Maass† $(1998)^{48}$ & $70 v 17$ (coronary atherectomy, failed grafts and other vessels) & $30 \%$ v $0 \%$ & & $16 \% \ddagger v$ not done \\
\hline Wong $(1998)^{54}$ & $58 v 58$ (coronary atherectomy and failed grafts) & $39 \%$ v $12-30 \%$ & & \\
\hline Shor $(1992)^{66}$ & $10 v 5$ (coronary artery) Cases were highly selected from 1000 arteries & & $5 / 7(71 \%) v 0 / 5(0 \%)$ & $10 / 10 \S v$ not done \\
\hline Kuo $(1993)^{67}$ & $20 v 4$ (aortic atheroma) & & $30 \%$ v $0 \%$ & \\
\hline Chiut $(1997)^{58}$ & 76 ข 20 (carotid and aortic tissue) & & $71 \%$ v $0 \%$ & \\
\hline Muhlestein $†(1996)^{68}$ & 90 coronary atherectomies 24 other controls & & & $79 \% v 4.2 \%$ \\
\hline \multicolumn{5}{|l|}{ Studies without controls } \\
\hline Kuo $(1993)^{60}$ & 36 (coronary artery) & $13 / 30(43 \%)$ & $15 / 36(42 \%)$ & $6 / 21 \rrbracket(29 \%)$ \\
\hline Campbell $(1995)^{59}$ & 37 (coronary atherectomy) & $32 \%$ & $45 \%$ & $2 / 25$ \\
\hline Ramirez $(1996)^{46}$ & 12 (coronary artery) & $41.7 \%$ & $41.7 \%$ & $8 \% \ddagger 25 \% \rrbracket$ \\
\hline Jackson $(1997)^{47}$ & 25 (carotid endarterectomy) & $24 \%$ & $8 / 16(50 \%)$ & $4 \% \ddagger$ \\
\hline Blasi $(1996)^{69}$ & $51(\mathrm{AAA})$ & $51 \%$ & & \\
\hline Weiss $(1996)^{18}$ & 72 (coronary atherectomies) & $1 / 50(2 \%)$ & & $0 / 22 \neq \S$ \\
\hline Davidson $(1998)^{56}$ & 60 (coronary artery) & $14 / 60(23.3 \%)$ & $20 / 60(33.3 \%)$ & \\
\hline Bauriedel $(1998)^{57}$ & 32 (carotid and coronary arteries) & & $47 \%$ & \\
\hline Lindholt $(1998)^{52}$ & 20 (AAA) & $0 \%$ & & \\
\hline Paterson $(1998)^{53}$ & 30 (carotid and coronary arteries) & $0 \%$ & & \\
\hline Saldeen $(1998)^{45}$ & 60 (coronary arteries) & & $62 \%$ & \\
\hline
\end{tabular}

${ }^{\star}$ In some cases, more than one specimen was obtained from each patient. In these cases and where it was possible to determine from the papers, results are expressed as positive patients per number of patients.

†Studies where control vessels did not come from age matched subjects or where control subjects were poorly described; $\ddagger$ culture; $\$$ electron microscopy; $\uparrow$ direct immunofluorescence.

PCR, polymerase chain reaction; ICC, immunocytochemistry; AAA, abdominal aortic aneurysm; IMA, internal mammary artery. 
that chronic infection occurs. We also used the Stary grade to assess coronary atherosclerosis and found no association of severity with the presence of $C$ pneumoniae. ${ }^{71}$ Nevertheless, in another study, $C$ pneumoniae was found in diseased segments of carotid artery but not in adjacent segments that were macroscopically normal but had early disease. ${ }^{50}$ Similarly, in a study of young adults, $C$ pneumoniae was more common in arteries with atheroma than in arteries with intimal thickening, but was absent in arteries that appeared normal. ${ }^{51}$

Few studies have examined the extent of $C$ pneumoniae infection in individuals. In a postmortem study where two segments from each coronary artery were examined, we found that in patients with three vessel coronary artery disease, the organism was just as likely to be found in one as in two or three arteries. ${ }^{71}$ Similarly, another study found that contiguous segments from diseased coronary arteries or different coronary arteries from the same subject were not all positive for $C$ pneumoniae. ${ }^{46}$

In summary, pathological studies have shown that $C$ pneumoniae is common in atherosclerotic vessels from a wide variety of sources, including those from young subjects. ${ }^{51}{ }^{72}$ This would suggest that chronic infection occurs. $C$ pneumoniae may be more prevalent in severe compared with mild lesions but its distribution in an individual often does not necessarily match that of atherosclerosis. These findings suggest that $C$ pneumoniae exacerbates rather than causes atherosclerosis. Thus, one study using ICC and electron microscopy reported that it was more frequently found in atherectomy samples from patients presenting with acute coronary syndrome than from patients with stable angina.$^{57}$ However, we found using PCR that $C$ pneumoniae was just as likely to be found in mildly diseased lesions as in fatal plaques with acute thrombosis, rupture, or haemorrhage. ${ }^{71}$ Also, $C$ pneumoniae has been found in vessels not usually associated with atherosclerosis, such as the internal mammary artery and saphenous vein. ${ }^{54} 72$

In vitro, animal, and antibiotic studies Evidence of the potential pathogenicity of $C$ pneumoniae for vasculature comes from four studies of experimental $C$ pneumoniae infection in the New Zealand white rabbit. ${ }^{73-76}$ These rabbits do not spontaneously develop atherosclerosis until a late stage, but may do so if given cholesterol supplemented diets. Such a model is useful in assessing whether $C$ pneumoniae exacerbates or causes atherosclerosis. Intranasal inoculation with $C$ pneumoniae in rabbits fed a normal diet was found to induce aortic inflammatory changes consistent with early atherosclerosis, ${ }^{75}$ including foam cells, ${ }^{74}$ in two studies but not in a third. ${ }^{73}$ The histological changes were early and compatible with inflammation. In the largest study, in rabbits fed a diet supplemented with small amounts of cholesterol, lesion size was significantly increased in infected compared with uninfected rabbits, and these changes could be prevented by azithromycin. ${ }^{76}$
At the cellular level, recent studies indicate how $C$ pneumoniae may interact with known risk factors of atherosclerosis to cause atheroma. It is clear that $C$ pneumoniae is able to infect most of the cell types involved in atherogenesis, including endothelium, smooth muscle cells, and macrophages. ${ }^{77}$ Intriguingly, a recent study found that human macrophages infected with $C$ pneumoniae and incubated with low density lipoprotein were transformed into foam cells, the characteristic cells of early atheroma, within 22 hours. $^{78}$

A key question is whether $C$ pneumoniae plays a unique role in the induction of atherosclerosis. Other infectious agents, including Helicobacter pylori, periodontal bacteria, and cytomegalovirus, have been implicated. ${ }^{43}$ All bacteria, including chlamydia, share highly homologous heat shock proteins (HSP) capable of inducing autoimmune antibodies in humans caused by the presence of cross reactive sites on human HSP. Macrophages in atherosclerotic lesions express high concentrations of HSP, ${ }^{79}$ and concentrations of specific antibodies to HSP are increased in the sera of patients with atherosclerosis. ${ }^{15}$ One possibility is that the immune response to bacterial infection generally may interact with chlamydial or human HSP in vessel walls, so exacerbating atherosclerosis. A recent study found that chlamydial and human HSP-60 co-localises in atherosclerotic lesions and that both types of HSP could induce $\mathrm{TNF}_{\alpha}$ (tumour necrosis factor) and matrix degrading metalloproteinase activity when incubated with mouse macrophages. $^{80}$

There have been two small antibiotic trials where patients with unstable angina or myocardial infarction were given macrolide antibiotics and were followed up. ${ }^{81}$ Both reported beneficial effects in terms of reduction of further coronary events. A third study gave doxycycline to men who had had previous coronary artery bypass graft surgery. ${ }^{83}$ No change in antibody titres or basal nitric oxide production was seen after four months of treatment. Preliminary reports from a fourth antibiotic trial reported no reduction in further coronary events ${ }^{84}$ Larger studies with longer follow up periods are needed; such trials are either underway or being planned. However, there is debate as to whether $C$ pneumoniae can be eradicated and how long treatment should be. One study is planning to give antibiotics for a year. ${ }^{85}$ Macrolide antibiotics have antiinflammatory as well as antimicrobial properties and these will need to be controlled for. Pending the results of such trials, antibiotic therapy is not indicated.

\section{Conclusion}

There is as yet no conclusive evidence that $C$ pneumoniae causes atherosclerosis or precipitates acute coronary syndromes. Current knowledge about its distribution in blood vessels, animal studies, and antibiotic trials suggest that it may exacerbate atherosclerosis but these are preliminary results. There is no doubt that $C$ pneumoniae is commonly found in blood vessels and it is important to discover 
what the effects of infection are. It is too early to know whether antibiotics will play a role but such studies should be targeted at those who are clearly infected. Serology is an unreliable and poorly standardised surrogate for infection, failing in some studies to discriminate between those subjects with and without $C$ pneumoniae. ${ }^{50}{ }^{58}$ This may be because seropositive subjects in whom $C$ pneumoniae was not found had the organism at sites other than those investigated. However, it is clear that $C$ pneumoniae infection may occur in the presence of low specific antibody levels. ${ }^{596}$ It has been reported from Malaysia that $C$ pneumoniae can be detected by PCR in serum samples, ${ }^{86}$ although this has not been confirmed by other studies. ${ }^{87}$ A promising possibility is the finding that $C$ pneumoniae DNA can be detected in circulating white cells by PCR. ${ }^{27} 88$ Using this technique, we have found in Southampton an association of coronary artery disease with circulating $C$ pneumoniae DNA in men but not women. ${ }^{83}$ In northern Sweden, in a population with a high seroprevalence of $C$ pneumoniae antibody, a high prevalence of circulating $C$ pneumoniae DNA was found in both patients and blood donors. ${ }^{27}$

The potential role of $C$ pneumoniae and other infectious agents in coronary artery disease is an exciting and crucial area of cardiac research. Our hypothesis is that infection plays a role in only a subset of patients with coronary artery disease. Nevertheless the impact on public health could be substantial and the challenge to researchers is how to identify those patients most at risk.

Collaborative research in Southampton between molecular microbiology, experimental pathology and the Wessex cardiothoracic units is supported by the British Heart Foundation and the Wessex Cardiac Trust to whom we are most grateful.

1 Grayston JT, Kuo CC, Wang SP, et al. A new Chlamydia psittaci strain, TWAR, isolated in acute respiratory tract infections. $N$ Engl $\mathcal{F}$ Med 1986;315:161-8.

2 Aldous MB, Grayston JT, Wang SP, et al. Seroepidemiology of Chlamydia pneumoniae TWAR infection in Seattle of Chlamydia pneumoniae TWAR infection in

3 Kanamoto Y, Ouchi K, Mizui M, et al. Prevalence of antibody to Chlamydia pneumoniae TWAR in Japan. 7 Clin Microbiol 1991;29:816-18.

4 Einarsson S, Sigurdsson HK, Magnusdottir SD, et al. Age specific prevalence of antibodies against Chlamydia pneumoniae in Iceland. Scand F Infect Dis 1994; 26:393-7.

5 Karvonen M, Tuomilehto J, Pitkaniemi J, et al. Chlamydia pneumoniae IgG antibody prevalence in south western and eastern Finland in 1982 and 1987. Int f Epidemiol 1994;23: 176-84.

6 Patnode D, Wang SP, Grayston JT. Persistence of Chlamydia pneumoniae, strain TWAR micro-immunofluorescent antibody. In: Chlamydial infections. Bowie WR, Caldwell HD, Jones RP, et al, eds. Cambridge: Cambridge University Press, 1990:406-9.

7 Grayston JT, Mordhorst C, Bruu AL, et al. Countrywide epidemics of Chlamydia pneumoniae, strain TWAR, in epidemics of Chlamydia pneumoniae, strain TWAR, in

8 Karvonen M, Tuomilehto J, Pitkaniemi J, et al. The Karvonen M, Tuomilehto J, Pitkaniemi J, et al. The ern Finland, 1972-1987. Epidemiol Infect 1993;110:34960.

9 Grayston JT. Chlamydia pneumoniae, strain TWAR. Chest 1989;95:664-9.

10 Chirgwin K, Roblin PM, Gelling M, et al. Infection with Chlamydia pneumoniae in Brooklyn. F Infect Dis 1991;163 757-61.

11 Hyman CL, Augenbraun MH, Roblin PM, et al. Asymptomatic respiratory tract infection with Chlamydia pneumoniae TWAR. 7 Clin Microbiol 1991;29:2082-3.

12 Kern DG, Neil MA, Schachter J. A seroepidemiologic study of Chlamydia pneumoniae in Rhode Island: evidence of of Chlamydia pneumoniae in Rhode Island: evider

13 Maurin M, Eb F, Etienne J, et al. Serological cross-reactions between Bartonella and Chlamydia species: implications for diagnosis. F Clin Microbiol 1997;35:2283-7.
14 Domeika M, Domeika K, Paavonen J, et al. Humoral immune response to conserved epitopes of Chlamydia trachomatis and human 60-kDa heat-shock protein in women with pelvic inflammatory disease. F Infect Dis 1998;177: $714-19$.

15 Xu Q, Willeit J, Marosi M, et al. Association of serum antibodies to heat shock protein 65 with carotid atherosclerosis. Lancet 1993;341:225-9.

16 Leinonen M, Linnanmaki E, Mattila $\mathrm{K}$, et al. Circulating immune complexes containing chlamydial lipopolysaccharide in acute myocardial infarction. Microb Pathog 1990;9: ride in 673 .

17 Leinonen M, Mattila K, Kohlmeier L, Saikku P. Chlamydia pneumoniae specific antibodies and immune complexes in German patients with acute myocardial infarction. In: Orfila J, Byrne GI, Chernesky M, et al. eds. Chlamydial infections. Proceedings of the eighth international symposium on human chlamydial infections. Bologna: Societa Editrice Esculapio, 1994:209-11.

18 Weiss SM, Roblin PM, Gaydos CA, et al. Failure to detect Chlamydia pneumoniae in coronary atheromas of patients undergoing atherectomy. F Infect Dis 1996;173:957-62.

19 Saikku P, Leinonen M, Tenkanen L, et al. Chronic Chlamydia pneumoniae infection as a risk factor for coronary heart disease in the Helsinki heart study. Ann Intern Med 1992;116:273-8.

20 Miettinen H, Lehto S, Saikku P, et al. Association of Chlamydia pneumoniae and acute coronary heart disease events in non-insulin dependent diabetic and non diabetic subjects in Finland. Eur Heart F 1996;17:682-8.

21 Diedrichs H, Schneider CA, Scharkus S, et al. Prevalence of chlamydia antibodies in patients with coronary heart disease. Herz Kreislauf 1997;29:304-7.

22 Halme S, Syrjala H, Bloigu A, et al. Lymphocyte responses to chlamydia antigens in patients with coronary heart disease. Eur Heart $\mathcal{F}$ 1997;18:1095-101.

23 Saikku P, Mattila K, Nieminen MS, et al. Serological evidence of an association of a novel Chlamydia, TWAR, with chronic coronary heart-disease and acute myocardialinfarction. Lancet 1988;II:983-986.

24 Cook PJ, Honeybourne D, Lip GYH, et al. Chlamydia pneumoniae antibody titers are significantly associated with acute stroke and transient cerebral ischemia - the west Birmingham stroke project. Stroke 1998;29:404-10.

25 Mazzoli S, Tofani N, Fantini A, et al. Chlamydia pneumoniae antibody response in patients with acute myocardial infarction and their follow-up. Am Heart $f$ 1998;135:15-20.

26 Kark JD, Leinonen M, Paltiel O, et al. Chlamydia pneumoniae and acute myocardial infarction in Jerusalem. Int $\mathcal{f}$ Epidemiol 1997;26:730-8.

27 Boman J, Soderberg S, Forsberg J, et al. High prevalence of Chlamydia pneumoniae DNA in peripheral blood mononuclear cells in patients with cardiovascular disease and in middle-aged blood donors. F Infect Dis 1998;178:274-7.

28 Blasi F, Cosentini R, Raccanelli R, et al. A possible association of Chlamydia pneumoniae infection and acute myocardial infarction in patients younger than 65 years of age. Chest 1997;112:309-12.

29 Ossewaarde JM, Feskens EM, DeVries A, et al. Chlamydia pneumoniae is a risk factor for coronary heart disease in symptom free elderly men, but Helicobacter pylori and symptom free elderly men, but Helicobacter pylori and

30 Nieto FJ, Folsom A, Sorlie P, et al. Chlamydia pneumonia infection and incident coronary heart disease: the atherosclerosis risk in communities (ARIC) study [abstract]. Am f Epidemiol 1997;145:331

31 Siscovick DS, Schwartz SM, Corey L, et al. Antibody to Chlamydia pneumoniae, herpes simplex virus type 1 , cytomegalovirus and incident myocardial infarction and coronary heart disease death: the cardiovascular health study [abstract]. Circulation 1998;97:2.

32 Dahlen GH, Boman J, Birgander LS, et al. Lp (A) Lipoprotein, IgG, IgA and IgM antibodies to Chlamydia pneumoniae and HLA Class II genotype in early coronary artery niae and HLA Class II genotype in early
disease. Atherosclerosis 1995;114:165-74.

33 Mendall MA, Carrington D, Strachan D, et al. Chlamydia pneumoniae - risk factors for seropositivity and association with coronary heart disease. $\mathcal{F}$ Infect 1995;30:121-8

34 Thom DH, Wang SP, Grayston JT, et al. Chlamydia pneumoniae strain TWAR antibody and angiographically demonstrated coronary artery disease. Arterioscler Thromb 1991;11:547-51.

35 Thom DH, Grayston JT, Siscovick DS, et al. Association of prior infection with Chlamydia pneumoniae and angiographically demonstrated coronary artery disease. $¥ A M A$ 1992;268:68-72.

36 Melnick SL, Shahar E, Folsom AR, et al. Past infection by Chlamydia pneumoniae strain TWAR and asymptomatic carotid atherosclerosis. Am f Med 1993;95:499-504.

37 Patel P, Mendall MA, Carrington D, et al. Association of Helicobacter pylori and Chlamydia pneumoniae infections with coronary heart disease and cardiovascular risk factors. BMF 1995;311:711-14.

38 Thomas GN, Scheel O, Koehler AP, et al. Respiratory chlamydial infections in a Hong Kong teaching hospital and association with coronary heart disease. Scand F Infect Dis 1997;104(suppl):30-3.

39 Toss H, Gnarpe J, Gnarpe H, et al. Increased fibrinogen levels are associated with persistent Chlamydia pneumoniae infection in unstable coronary artery disease. Eur Heart $\mathcal{F}$ 1998;19:570-7. 
40 Wimmer MLJ, Sandmannstrupp R, Saikku P, et al. Association of chlamydial infection with cerebrovascular Association of chlamydial infect
disease. Stroke 1996;27:2207-10.

41 Linnanmaki E, Leinonen $\mathrm{M}$, Mattila $\mathrm{K}$, et al. Chlamydia pneumoniae specific circulating immune-complexes in patients with chronic coronary heart disease. Circulation 1993;87:1130-4

42 Anderson JL, Carlquist JF, Muhlestein JB, et al. Evaluation of C-reactive protein, an inflammatory marker, and infectious serology as risk factors for coronary artery disease and myocardial infarction. f Am Coll Cardiol 1998; 32:35-41

43 Danesh J, Collins R, Peto R. Chronic infections and coronary heart disease: is there a link? Lancet 1997;350:430-6.

44 Salomaa V, Arstila M, Kaarsalo E, et al. Trends in the incidence of and mortality from coronary heart disease in Finland, 1983-1988. Am f Epidemiol 1992;136:1303-15.

45 Saldeen TP, Ericson K, Lindquist O, et al. Chlamydia and HLA-DR genotypes in coronary atherosclerosis [abstract]. f Am Coll Cardiol 1998;31:A272.

46 Ramirez JA, Ahkee S, Summersgill JT, et al. Isolation of Chlamydia pneumoniae from the coronary-artery of a patient with coronary atherosclerosis. Ann Intern Med 1996;125:979

47 Jackson LA, Campbell LA, Kuo CC, et al. Isolation of Chlamydia pneumoniae from a carotid endarterectomy specimen. F Infect Dis 1997;176:292-5.

48 Mass M, Bartels C, Engel PM, et al. Endovascular presence of viable Chlamydia pneumoniae is a common phenomenon in coronary artery disease. 7 Am Coll Cardiol 1998;31: 827-32.

49 Tejada C, Strong JP, Montenegro MR, et al. Distribution of coronary and aortic atherosclerosis by geographic location, race, and sex. Lab Invest 1968;18:509-26.

50 Maass M, Krause E, Engel PM, et al. Endovascular presence of Chlamydia pneumoniae in patients with hemodynamically effective carotid artery stenosis. Angiology 1997;48: 699-706.

51 Kuo CC, Grayston JT, Campbell LA, et al. Chlamydia pneumoniae (TWAR) in coronary-arteries of young-adults
(15-34 years old). Proc Natl Acad Sci USA 1995;92:691114.

52 Lindholt JS, Ostergard L, Henneberg EW, et al. Failure to demonstrate Chlamydia pneumoniae in symptomatic abdominal aortic aneurysms by a nested polymerase chain reaction (PCR). Eur $\mathcal{F}$ Vasc Endovasc Surg 1998;15:161-4.

53 Paterson DL, Hall J, Rasmussen SJ, et al. Failure to detect Chlamydia pneumoniae in atherosclerotic plaques of Australian patients. Pathology 1998;30:169-72.

54 Wong Y, Thomas M, Gallagher PJ, et al. The prevalence of Chlamydia pneumoniae in atherosclerotic and normal blood vessels of patients undergoing redo and first time coronary artery bypass graft surgery [abstract]. $\mathcal{F} \mathrm{Am}$ Coll Cardiol 1998;31:A146.

55 Ong G, Thomas BJ, Mansfield AO, et al. Detection and widespread distribution of Chlamydia pneumoniae in the vascular system and its possible implications. F Clin Pathol vascular system

56 Davidson M, Kuo CC, Middaugh JP, et al. Confirmed previous infection with Chlamydia pneumoniae (TWAR) and its presence in early coronary atherosclerosis. Circulation 1998;98:628-33.

57 Bauriedel G, Schmucking I, Hutter R, et al. Evidence of Chlamydia pneumoniae in human arteriosclerotic lesions: Insights into its possible pathogenic role [abstract]. $\mathcal{F} \mathrm{Am}$ Coll Cardiol 1998;31:A390-1.

58 Chiu B, Viira E, Tucker W, et al. Chlamydia pneumoniae, cytomegalovirus, and herpes simplex virus in atherosclerosis of the carotid artery. Circulation 1997;96:2144-8.

59 Campbell LA, OBrien ER, Cappuccio AL, et al. Detection of Chlamydia pneumoniae TWAR in human coronary atherectomy tissues. F Infect Dis 1995;172:585-8.

60 Kuo CC, Shor A, Campbell LA, et al. Demonstration of Chlamydia pneumoniae in atherosclerotic lesions of coronary arteries. F Infect Dis 1993;167:841-9.

61 Petersen E, Boman J, Persson K, et al. Chlamydia pneumoniae in human abdominal aortic aneurysms. Eur 7 Vasc Endovasc Surg 1998;15:138-42.

62 Grayston JT, Kuo CC, Coulson AS, et al. Chlamydia pneumoniae (TWAR) in atherosclerosis of the carotid-artery. Circulation 1995;92:3397-400.

63 Jackson LA, Campbell LA, Schmidt RA, et al. Specificity of detection of Chlamydia pneumoniae in cardiovascular atheroma-evaluation of the innocent bystander hypothesis. Am f Pathol 1997;150:1785-90.

64 Juvonen JJ, Tuvonen T, Laurila A, et al. Demonstration of Chlamydia pneumoniae in the walls of abdominal aortic aneurysms. $\mathcal{F}$ Vasc Surg 1997;25:499-505.

65 Kuo CC, Coulson AS, Campbell LA, et al. Detection of Chlamydia pneumoniae in atherosclerotic plaques in the walls of arteries of lower extremities from patients undergoing bypass operation for arterial obstruction. $\mathcal{F}$ Vasc Surg 1997;26:29-31

66 Shor A, Kuo CC, Patton DL. Detection of Chlamydia pneumoniae in coronary arterial fatty streaks and atheropneumoniae in coronary arterial fatty streaks
matous plaques. $S$ Afr Med $\mathcal{f} 1992 ; 82: 158-61$
67 Kuo CC, Gown AM, Benditt EP, et al. Detection of Chlamydia pneumoniae in aortic lesions of atherosclerosis by immunocytochemical stain. Arterioscler Thromb 1993; 13:1501-4.

68 Muhlestein JB, Hammond EH, Carlquist JF, et al. Increased incidence of Chlamydia species within the coronary arteries of patients with symptomatic atherosclerotic versus other forms of cardiovascular disease. $7 \mathrm{Am}$ Coll Cardiol 1996;27:1555-61.

69 Blasi F, Denti F, Erba M, et al. Detection of Chlamydia pneumoniae but not Helicobacter pylori in atherosclerotic plaques of aortic aneurysms. F Clin Microbiol 1996;34: plaques

70 Mehta JL, Saldeen TP, Rand K. Interactive role of infection, nflammation and traditional risk factors in atherosclerosis and coronary artery disease. F Am Coll Cardiol 1998;31: $1217-25$.

71 Thomas M, Wong YK, Thomas D, et al. Direct detection of C pneumoniae DNA at multiple sites in human coronary arteries post mortem and its relationship to the histological severity (Stary grade) of associated atherosclerotic plaque. In: Stephens RS, Byrne GI, Christiansen G, et al, eds. Chlamydial infections. Proceedings of the ninth international symposium on human chlamydial infection. Internation
Chlamydia Symposium, San Francisco, 1998:199-202.

72 TaylorRobinson D, Ong G, Thomas BJ, et al. Chlamydia pneumoniae in vascular tissues from heart-transplant donors. Lancet 1998;351:1255.

73 Moazed TC, Kuo CC, Patton DL, et al. Experimental rabbit models of Chlamydia pneumoniae infection. Am $\mathcal{F}$ Pathol 1996;148:667-76.

74 Fong IW, Chiu B, Viira E, et al. Rabbit model for Chlamydia pneumoniae infection. F Clin Microbiol 1997;35:48-52.

75 Laitinen K, Laurila A, Pyhala L, et al. Chlamydia pneumoniae infection induces inflammatory changes in the aortas of rabbits. Infect Immun 1997;65:4832-5.

76 Muhlestein JB, Anderson JL, Hammond EH, et al. Infection with Chlamydia pneumoniae accelerates the development of atherosclerosis and treatment with azithromycin prevents it in a rabbit model. Circulation 1998;97:633-6.

77 Gaydos CA, Summersgill JT, Sahney NN, et al. Replication of Chlamydia pneumoniae in-vitro in human macrophages, endothelial cells, and aortic artery smooth muscle cells. Infect Immun 1996;64:1614-20.

78 Kalayoglu MV, Byrne GI. Induction of macrophage foam cell formation by Chlamydia pneumoniae. $\mathcal{F}$ Infect Dis 1998;177:725-9.

79 Schett G, Metzler B, Mayr M, et al. Macrophage-lysis mediated by autoantibodies to heat shock protein $65 / 60$. Atherosclerosis 1997;128:27-38.

$80 \mathrm{Kol} \mathrm{A}$, Sukhova GK, Lichtman AH, et al. Chlamydial heat shock protein 60 localises in human atheroma and regulates macrophage tumor necrosis factor-alpha and matrix metalloproteinase expression. Circulation 1998;98: $300-7$.

81 Gupta S, Leatham EW, Carrington D, et al. Elevated Chlamydia pneumoniae antibodies, cardiovascular events, and azithromycin in male survivors of myocardial infarction. Circulation 1997;96:404-7.

82 Gurfinkel E, Bozovich G, Daroca A, et al. Randomised trial of roxithromycin in non Q-wave coronary syndromes: ROXIS pilot study. Lancet 1997;350:404-7.

83 Sinisalo J, Mattila K, Nieminen MS, et al. The effect of prolonged doxycycline therapy on Chlamydia pneumoniae serological markers, coronary heart disease risk factors and forearm basal nitric oxide production. $f$ Antimicrob Chemother 1998;41:85-92.

84 ACADEMIC (azithromycin in coronary artery disease: elimination of myocardial infection with chlamydia). Meeting report. Clin Cardiol 1998;21:429-30.

85 Grayston JT. Antibiotic treatment of Chlamydia pneumoniae for secondary prevention of cardiovascular events. Circulation 1998;97:1669-70.

86 Naidu BR, Ngeow YF, Kannan P, et al. Evidence of Chlamydia pneumoniae infection obtained by the polymerase chain reaction (PCR) in patients with acute myocardial infarction and coronary heart disease. $\mathcal{F}$ Infect 1997;35: 199-200.

87 Boman J, Petersen E, Persson K, et al. Failure to detect Chlamydia pneumoniae DNA by nested PCR in serum samples of patients with infrarenal abdominal aortic aneurysms. In: Stephens RS, Byrne GI, Christiansen G, et al, eds. Chlamydial infections. Proceedings of the ninth international symposium on human chlamydial infection. International Chlamydia Symposium, San Francisco, 1998: 211-14.

88 Wong YK, Dawkins K, Ward ME. Detection of C pneumoniae DNA circulating in the blood of patients attending for cardiac angiography: preliminary clinical and serological correlates. In: Stephens RS, Byrne GI, Christiansen G, et al, eds. Chlamydial infections. Proceedings of the ninth international symposium on human chlamydial infection. International Chlamydial Symposium, San Francisco, 1998:227-30 